\title{
Pisat's Visual Vasopressor Injection Needle: A New Instrument for enhancing Patient Safety in Laparoscopic Myomectomy
}

\section{Sanket Pisat}

\begin{abstract}
The use of vasoconstrictor agents like vasopressin during laparoscopic myomectomy reduces blood loss during surgery and reduces operative time. However, certain side effects related to the cardiovascular system have also been reported with its use. These side effects could possibly be due to inadvertent intravascular injection of the drug. The author aims to describe a new design of an injection needle, Pisat's Visual Vasopressor Injection Needle (VVIN) that can be used during laparoscopic myomectomy to reduce the likelihood of an inadvertent intravascular injection of a vasoconstrictor solution.
\end{abstract}

Keywords: Fibroid, Laparoscopy, Myomectomy, Vasopressin.

How to cite this article: Pisat S. Pisat's Visual Vasopressor Injection Needle: A New Instrument for enhancing Patient Safety in Laparoscopic Myomectomy. Int J Gynecol Endsc 2018;2(1):4-5.

Source of support: Nil

Conflict of interest: None

\section{INTRODUCTION}

It is common practice during laparoscopic myomectomy to inject a diluted solution of a vasopressor agent into the fibroid to reduce intraoperative blood loss. ${ }^{1}$ The use of such vasoconstrictive agents has also been associated with intraoperative hemodynamic fluctuations, ${ }^{2}$ and very occasionally may also cause severe morbidity or mortality. These side effects could be due to its inadvertent injection into the blood vessels over the fibroid. These side effects have resulted in the drug not being commercialized in some countries across the world.

\section{SUMMARY}

The standard laparoscopic myomectomy needle is about $33 \mathrm{~cm}$ in length and is completely opaque. Predominantly, the fibroid vasculature consists of thin-walled capillaries

\section{Consultant Gynaec Endoscopic Surgeon}

Department of Minimal Access Surgery, Akanksha Hospital Manish Tower, 4 Bungalows, Mumbai, Maharashtra, India

Corresponding Author: Sanket Pisat, Department of Minimal Access Surgery, Akanksha Hospital Manish Tower, 4 Bungalows Mumbai, Maharashtra, India, Phone: +919967710063, e-mail: sanket.pisat@gmail.com and small veins. ${ }^{3}$ Even if the surgeon does mistakenly puncture a blood vessel, the column of blood is extremely unlikely to rise to a distance high enough to discolor the fluid in the syringe red.

By adding a small, transparent area at the proximal end of the instrument, a blood-stained aspirate can be immediately seen in case the needle has punctured a vessel. ${ }^{4}$ The needle can then be repositioned at another site over the fibroid. This significantly reduces the possibility of injecting the vasopressor solution in the vascular channels over the fibroid.

\section{DETAILED DESIGN OF PISAT'S VVIN}

The VVIN is a $33 \mathrm{~cm}$ long and $5 \mathrm{~mm}$ wide hollow needle, similar to the existing needle that is used for injecting vasopressin solution during laparoscopic myomectomy. At one end of the instrument, which remains outside the abdomen, is a Luer lock hub that attaches to a standard syringe. At the other end, which is inserted into the abdomen, the VVIN has a disposable and detachable tip which can be screwed on to the main shaft (Fig. 1).

\section{THE VVIN TIP}

The disposable VVIN tip has a $1 \mathrm{~cm}$ long transparent plastic cylindrical body and a $2.5 \mathrm{~cm}$ long metallic needle tip. The metallic shaft can be sterilized by autoclaving, and the transparent tip is disposable.

\section{USING THE VVIN}

The disposable tip is fixed over the shaft and the assembly is flushed with diluted vasopressin solution. After choosing a suitable site over the fibroid, as per the surgeon's preference, the VVIN is thrust into the subcapsular plane. On aspirating with a syringe, if the tip of the needle has penetrated a blood vessel, the plastic hub of the VVIN shows a blood-stained aspirate (Fig. 2). However, if the

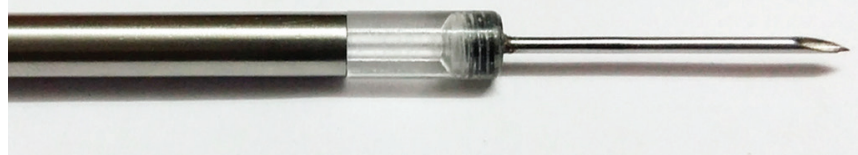

Fig. 1: Visual vasopressor injection needle 


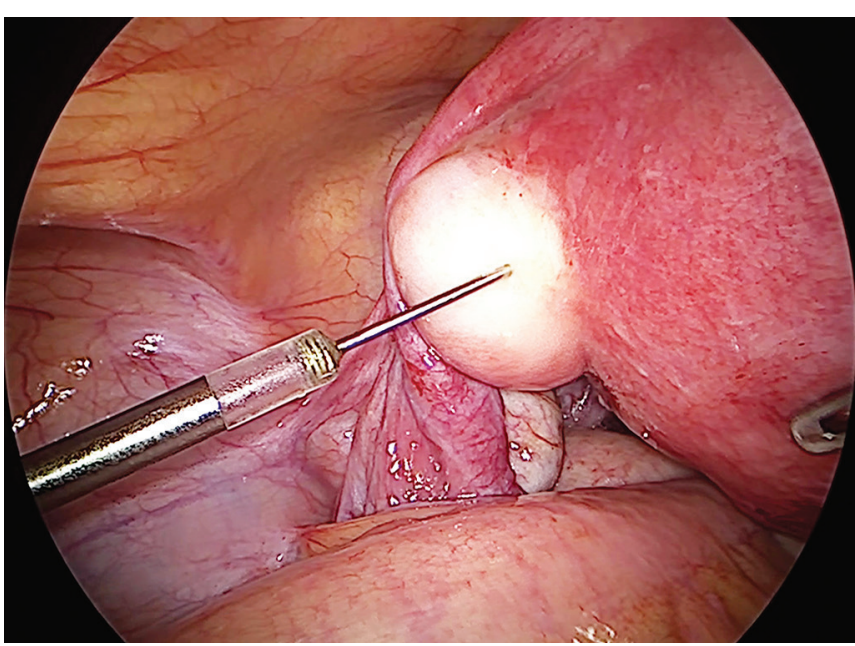

Fig. 2: Visual vasopressor injection needle injecting under the myoma capsule

needle is not within a vessel lumen, the aspiration shows no blood and vasopressin can be injected safely (Fig. 3).

\section{EFFICACY}

Using a VVIN, a volume of just $0.03 \mathrm{~mL}$ of blood is sufficient to detect a vascular puncture. This is lesser than one single drop of blood (approximately $0.05 \mathrm{~mL}$ ), and about 27 times more sensitive than a regular injection needle, which requires about $0.8 \mathrm{~mL}$ of blood to show up as a positive aspirate in the syringe. A prospective randomized study published by Pisat and van Herendael ${ }^{4}$ demonstrated significantly lesser fluctuations in pulse and blood pressure with the use of a VVIN as compared with a regular $5 \mathrm{~mm}$ myoma infiltration needle, with no adverse cardiovascular effects.

The VVIN has several other applications than laparoscopic myomectomy. It can be used for injecting vasopressin before adenomyoma removal, during laparoscopic ovarian cystectomy for endometriomas, in cesarean scar ectopic pregnancy for devascularizing the uterine myometrium, and also for salpingotomy in cases of ectopic pregnancy.

The likelihood of serious life-threatening side effects due to vasopressin injection during laparoscopic myomectomy is not very frequent. This may be because a diluted solution of vasopressin is used. (The author uses 20 units of vasopressin diluted in $200 \mathrm{~mL}$ of normal saline, at an effective concentration of $0.01 \mathrm{U} / \mathrm{mL}$ ). However, occasional catastrophic incidents have been reported even at low concentrations. These could be minimized or nullified completely by using a VVIN. This may be a

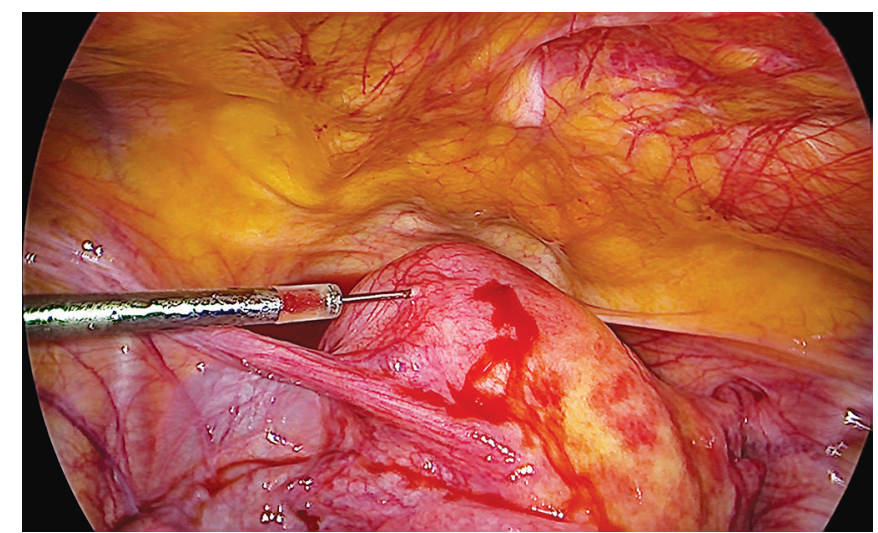

Fig. 3: The VVIN with blood seen in transparent tip

suitable alternative to stopping the usage of a relatively inexpensive drug that causes significant reduction in overall blood loss during surgery.

Using a VVIN would also provide documentary evidence that the surgeon has taken adequate care to avoid an intravascular injection, and thus may become a legal requirement in the future.

A patent application for the device has been filed both in India (Application no 201721001762) and internationally (PCT/IN/2017/050259). A worldwide analysis of patent records has confirmed that no similar instrument for use in laparoscopic myomectomy exists till date, making this invention a worldwide first. ${ }^{5}$ More details, case studies, and videos can be found online on www. pisatsvvin.com.

\section{REFERENCES}

1. Mettler L, Schollmeyer T, Tinelli A, Malvasi A, Alkatout I. Complications of uterine fibroids and their management, surgical management of fibroids, laparoscopy and hysteroscopy versus hysterectomy, haemorrhage, adhesions, and complications. Obstet Gynecol Int 2012 Apr;2012:791248.

2. Song T, Kim MK, Kim ML, Jung YW, Yun BS, Seong SJ. Use of vasopressin vs epinephrine to reduce haemorrhage during myomectomy: a randomized controlled trial. Eur J Obstet Gynecol Reprod Biol 2015 Dec;195:177-181.

3. Tinelli A. Uterine fibroid pseudocapsule: an update of its importance in fibroid management and female reproduction. Int J Reprod Biomed 2014;1(1):7-10.

4. Pisat S, van Herendael B. Pisat's visual vasopressor injection needle: an innovative tool for increasing patient safety in laparoscopic myomectomy. Surg Technol Int 2017 Jul;30: 197-204.

5. Pisat SV. Pisat's visual vasopressor injection needle: a new device for increasing patient safety in laparoscopic myomectomy. J Obstet Gynecol India 2017 Dec;67(6):451-453. 